\title{
Silylation of mica for lipase immobilization as biocatalysts in esterification
}

\begin{abstract}
Mica was modified either by acid treatment, grafting with aminopropyl-, octyl-, vinyl-, mercapto- and glycidoxy-triethoxysilanes, and activation of pre-treated support with glutaraldehyde (Glu). The derivatives were characterized by X-ray diffraction (XRD), infrared spectroscopy (FTIR), surface area and porosity analysis, scanning electron microscopy coupled with energy dispersive X-ray (SEM-EDX) and transmission electron microscopy (TEM) techniques. The modified micas were used for immobilization of lipase from Candida rugosa (CRL). Activity of the lipase was determined by esterification and exhibited the improved activity than the free enzyme following the order; Amino-CRLNGlu-AminoCRLNOctyl-CRLNVinyl-CRLNGlycidoxy CRLNMercapto-CRLNMica-CRL. Lipase immobilized mica showed enhanced protein loading(up to $8.22 \mathrm{mg}$ protein/g support) and immobilization (up to $78 \%$ ) compared to the free lipase and unmodified mica.
\end{abstract}

Keyword: Mica; Silanization; Immobilization; Candida rugosa lipase; Esterification 